\title{
GMR
}

\section{miR-71b regulation of insulin/IGF-1 signaling during starvation in planarians}

\author{
Y.Y. Wu ${ }^{1 *}$, J.M. Zhao ${ }^{1 *}$, Q. Liu', Q. Guo ${ }^{1}$, Z. Liu' ${ }^{1}$, X.X. Wang ${ }^{1}$, C.Y. Wang ${ }^{1}$, R.Y. Li', \\ Y.Z. Zhang ${ }^{1,2}$ and S.T. Zhang ${ }^{1,3}$ \\ 1School of Life Sciences, Zhengzhou University, Zhengzhou, Henan, China \\ ${ }^{2}$ Henan Key Laboratory of Bioactive Molecules, Zhengzhou University, Zhengzhou, \\ Henan, China \\ ${ }^{3}$ Collaborative Innovation Center of New Drug Research and Safty Evaluation, \\ Henan, China \\ ${ }^{*}$ These authors contributed equally to this study. \\ Corresponding author: S.T. Zhang / Y.Z. Zhang \\ E-mail: zhangst@zzu.edu.cn / yizhezhang@126.com \\ Genet. Mol. Res. 14 (4): 11905-11914 (2015) \\ Received February 20, 2015 \\ Accepted May 15, 2015 \\ Published October 5, 2015 \\ DOI http://dx.doi.org/10.4238/2015.October.5.4
}

\begin{abstract}
Planarians, which have a large population of stem cells called neoblasts, are molecularly tractable model systems used in the study of regeneration. However, planarians have strong resistance to hunger and have developed growth arrest strategies. For example, they can change their size and undergo growth regression during starvation periods. The results of the current study show that the microRNA, miR$71 \mathrm{~b}$, and the insulin/IGF-1 signaling pathway have important functions in the development of starvation-induced planarians. We demonstrate tissuespecific expression of miR-71b using in situ hybridization. By employing real-time polymerase chain reaction, we provide evidence that miR-71b is upregulated in starvation-induced planarians. Furthermore, we validate and verify the target genes of miR-71b.
\end{abstract}

Key words: Planaria; miR-71b; Starvation; Regeneration; Insulin/IGF-1 signaling pathway 


\section{INTRODUCTION}

Planarians (Platyhelminthes, Turbellaria) have extremely strong regeneration abilities and can grow into a complete individual when cut (transverse, longitudinal, or oblique cutting) (Newmark and Sánchez Alvarado, 2002). The strong regeneration ability of planarians is attributed to their large population of stem cells, called neoblasts. These cells are highly undifferentiated and account for 25 to $30 \%$ of the total cells of the individual. Neoblasts, which are the only mitotic cells in planarians, enable differentiation of cells into skin, muscles, nerve cells, and even reproductive cells (Baguñà et al., 1989; Wagner et al., 2011). Neoblasts also have the ability to resist starvation.

Planarians undergo a state of starvation during changes in environmental conditions. Dugesia japonica changes its size during periods of starvation, not because of changes in cell size, but because of a reduction in the number of neoblasts during cell division. Degenerative growth includes a reduction in size without damaging morphology and function. Planarians have a strong tolerance to starvation, can survive for three months without food, and can return to their normal state after acquiring food.

Studies have shown that planarians reshape their bodies during starvation periods. The microRNA (miRNA), miR-71, has a significant function in development during starvation of Caenorhabditis elegans, when its expression is upregulated. This gene inhibits the insulin signaling pathway or those parallel to the signaling pathway of gene expression. In addition, miR71 is an effective regulator of the long-term survival and recovery of $C$. elegans from starvationinduced L1 diapause because of the functional relationship of the gene with the insulin/IGF-1 signaling (IIS) pathway (Michelangela et al., 2003; Sri Devi et al., 2009). We utilized TargetScan and mirWIP to predict the target genes of miR-71 in D. japonica. The 3'-noncoding region of the phosphatidylinositol 3-kinase (age-1) gene and the 5'-end of the miR-71 seed region were complementary paired. Experimental results have shown that the age-1 gene, a crucial gene in the IIS pathway, is the target of miR-71. The daf-16 gene (a fork transcription factor) is located downstream of the IIS pathway (Lewis et al., 2005). By using the search function of miRBase, we determined that miR-71b in planarians and miR-71 in C. elegans are members of the miRNA family. These genes have identical seed regions that anchor the miRNA to the mRNA target. We conclude that miR-71b has important functions in the starvation-induced $D$. japonica.

The IIS pathway is conserved and has critical functions in the control of longevity, development, and metabolism across phylogeny (Barbieri et al., 2003; Narasimhan et al., 2009). miR-71 and the insulin receptor/PI3K gene interact to promote longevity in animals. Therefore, studying the expression and function of miR-71b to explain gene expression regulation during starvation in planarians, and to analyze interactions with the insulin receptor/PI3K gene, is of great significance. In the current study, we investigated whether expression of miR-71b changed in starvation-induced $D$. japonica and determined the effect of this gene on starvation-induced planarians.

\section{MATERIAL AND METHODS}

\section{Experimental material}

D. japonica (GI strain) asexual specimens were cultured in deionized water at $19^{\circ} \mathrm{C}$ and 
fed earthworms twice a week. The planarians were starved for one week before all experiments and their lengths ranged between 2 and $4 \mathrm{~mm}$ for in situ hybridization.

\section{RNA isolation and identification}

The planarians were starved for $0 \mathrm{~h}$, and 7,14 , and 21 days to determine the changes in miR-71b expression with time. At each time point, seven worms were collected and total RNA was immediately isolated from the starved planarians using TRIzol ${ }^{\circledR}$ Reagent (Invitrogen Life Technologies, Shanghai, China) in accordance with the manufacturer protocol.

\section{Confirmation of the expression profile over time}

We used miRNA real-time polymerase chain reaction (PCR) validation of miR-71b and its target genes to determine the genetic changes in $D$. japonica during various periods of starvation. We verified the role of this gene during starvation and the regularity of target gene expression to confirm their regulating relationship. Time expression profiles were used to study miR-71b and its target genes in the regeneration of planarians and to determine the relationship between the properties of neoblasts.

Stem-loop quantitative real-time PCR (qRT-PCR) was used to test and measure miRNA expression levels and to detect new miRNA (Livak and Schmittgen, 2001; Chen et al., 2005). This method was performed using an Eppendorf PCR detection system with SYBR Universal PCR master mix and the assays-on-demand gene expression products of the planarian target genes along with the endogenous controls, $\beta$-actin and $D j e f_{2}$. qRT-PCR was employed to analyze the expression of miR-71b on the basis of the endogenous miR-71c RNA content. qPCR data can be analyzed using absolute and relative quantification. In this study, we employed relative quantification PCR and used the $2^{-\triangle \Delta C T}$ method (Livak and Schmittgen, 2001) to analyze the relative genetic changes and expression of miR-71b. Experimental data are reported as means $\pm S D$ in triplicate independent samples.

The volume of the miRNA reverse transcription system was $20 \mu \mathrm{L}$ and the program specifications were set at $42^{\circ} \mathrm{C}$ for $15 \mathrm{~min}$ and $85^{\circ} \mathrm{C}$ for $5 \mathrm{~s}$. The volume of the real-time fluorescent qPCR system was $20 \mu \mathrm{L}$ and response procedures were set at $95^{\circ} \mathrm{C}$ for $3 \mathrm{~min}, 95^{\circ} \mathrm{C}$ for $10 \mathrm{~s}, 60^{\circ} \mathrm{C}$ for $20 \mathrm{~s}, 72^{\circ} \mathrm{C}$ for $20 \mathrm{~s}$, and $78^{\circ} \mathrm{C}$ for $20 \mathrm{~s}$ (fluorescence collection) for 40 cycles. Each sample was analyzed in three parallel experiments for all reactions.

\section{Tissue-specific expression analysis}

We performed whole-mount in situ hybridization (WISH) on planarians to analyze tissuespecific expression of miR-71b and to confirm the regulating relationship between miR-71b and its target genes in starvation-induced planarians. Two planarian groups, experimental and control, were established. Each group consisted of 15 worms in a 1.5-mL Eppendorf tube. The samples were viewed through the Mivnt image analysis system. We chose the modified locked nucleic acid (LNA) oligonucleotide for this experiment (Koshkin et al., 1998; Obika et al., 1998; Braasch and Corey, 2001; Petersen and Wengel, 2003). The LNA probe is fully complementary to miR-71b through Watson-Crick base pairing. The 5'-digoxigenin-labeled LNA-modified oligonucleotide was synthesized by Takara Biotechnology Co., Ltd. (Dalian, China). 


\section{Prediction of miR-71b target genes}

\section{Search for the target gene of miR-71b}

miRNAs interact with target mRNA to complete their biological functions. The search for miRNA target genes can be undertaken using two methods; biological experiment analysis or bioinformatic prediction. Here, we used bioinformatics analysis to predict the target genes of miR-71b.

The bioinformatic method is based on the algorithm of genetic samples that are screened and evaluated, and in the current study we used miRanda for the bioinformatic analysis of animal miRNA (Enright et al., 2003). RNA hybrid employs secondary structure analysis of the binding site of the miRNA and the target mRNA to predict the miRNA target by calculating the free energy of short chains of miRNA with the long chains (Rehmsmeier et al., 2004). PicTar has previously been used to predict the miRNA target genes of vertebrates, nematodes, and Drosophila (Krek et al., 2005), and TargetScan, which was developed by Lewis et al. in 2003, has been used for mammals. RNA22 is a miRNA target prediction software developed by Miranda et al. (2006). In the current study, the miRanda software was used to forecast the target genes using a process that is largely based on the complementary matching of miRNA and target mRNA 3' untranslated regions (UTRs), thermodynamic stability, and the target seed area conservative principles.

\section{Building the 3' UTR database for the planarians}

We downloaded the planarian sequence of miR-71b mRNA from NCBI and searched for the miR-71b full sequence mRNA 3' UTR to yield a 3' UTR database for planarians.

\section{Predicting miR-71b target genes using miRanda software}

We also utilized the $3^{\prime}$ UTR database and the target miRNA in the miRanda software. Prediction results show that the target sequence possesses the 5'-end 2-8 complete complementary sequence of the miRNA and that the target sequence and the miRNA secondary structure energy are $<14.00 \mathrm{kcal} / \mathrm{mol}$.

\section{miR-71b loss of function and validation of target gene expression}

Antagomir71b, complementary to miR-71b, was synthetized by Takara Biotechnology Co., Ltd. and diluted with RNase free water. The planarians were divided into experimental and control groups, with each group containing seven worms. The experimental group was injected with $100 \mathrm{mM}$ antagomir71b, whereas the control group was injected with RNase free water using a Drummond Nanoject II microinjector. Antagomir71b was injected into the planarians, except the pharynx and the head. Each group was injected every other day for a total of three injections at 32 $\mathrm{nL}$ dosages. The RNA isolation experiment was conducted for 6 to $8 \mathrm{~h}$ after completion of the last injection. Step-loop primers were used for the reverse transcription of miR-71b, and a quantitative primer for miR-71c was used for stable expression in the planarians. The quantitative primer pair used to predict the target genes of miR-71b was designed using Primer5.0 software (http://www. premierbiosoft.com/primerdesign/index.html). 


\section{RESULTS}

\section{miR-71b expression in starved planarians}

To assess the change in miR-71b expression in starvation-induced planarians, individuals starved for $0 \mathrm{~h}$ were used as the reference group. The expression of miR-71b differed at several starvation points. Inayswas downregulated ays. In individuals starved for 7 days, miR-71b expression was updownregulated (Figure 1), In planarians starved for 14 and 21 days, expression of miR-71b was upregulated in contrast to those starved for $0 \mathrm{~h}$, in which 7 days is downregulated most prominent and 14 days is upregulated most prominent and suggesting that this gene has an important function in starvation-induced planarians.

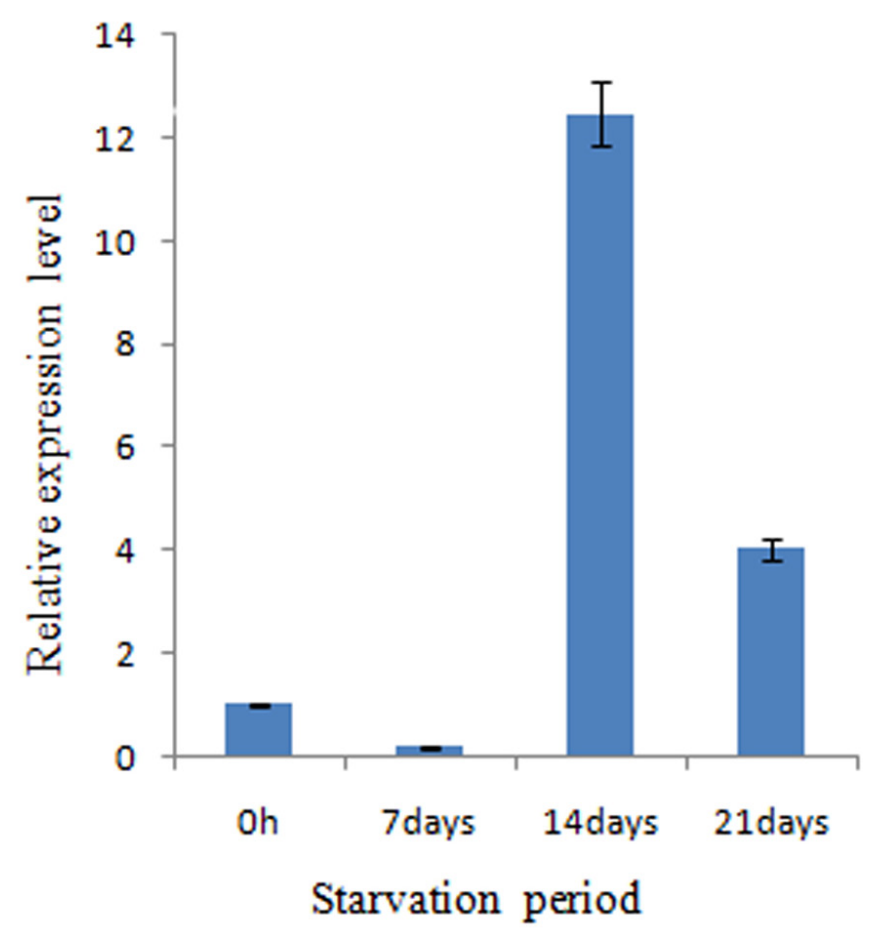

Figure 1. Relative expression of miR-71b in starvation-induced planarians following starvation for $0 \mathrm{~h}$ and 7,14 , and 21 days.

\section{miR-71b expression in planarians during in situ hybridization}

To analyze tissue-specific expression of miR-71b in planarians, we performed WISH. A digoxigenin-labeled antisense miR-71b probe was generated for miR-71b. Compared with the control, which showed no background hybridization with the hybridization solution (Figure 2A), miR-71b was significantly expressed in the pharynx and brain ganglia in planarians (Figure 2B). 

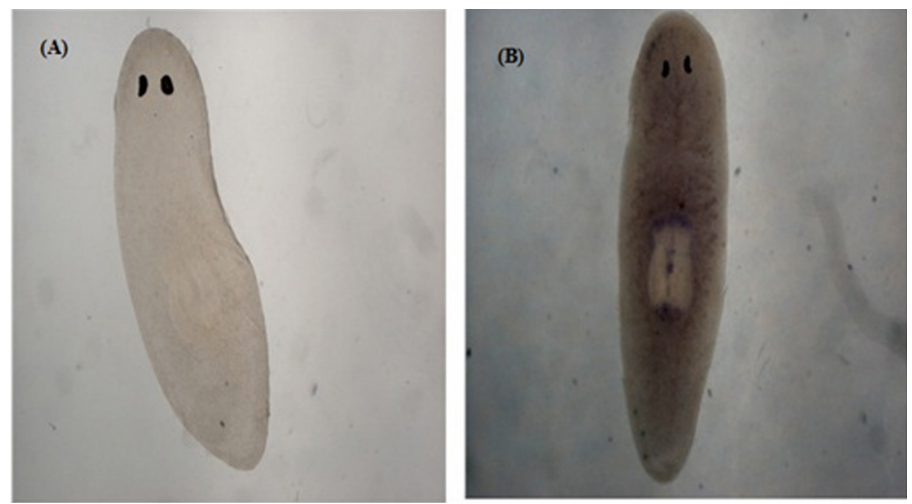

Figure 2. In situ hybridization experiment in planarians: A. control group (no expression) and B. experimental group, showing miR-71b expression in the parenchyma and brain ganglia.

\section{miR-71b gene expression}

To assess the ability of antagomir71b to specifically interfere with the function of miR$71 \mathrm{~b}$, we used the control group as the reference group and injected individuals in this group with RNase free water. The expression of miR-71b was downregulated 0.57 -fold upon injection with the antagomir71b solution compared with the control group (Figure 3 ).



Figure 3. Relative expression of miR-71b in planarians under control conditions (injected with RNase free water) and under loss of function conditions (experimental group, injected with antagomir71b solution). 


\section{Confirmation of miR-71b target genes}

We studied the function of miR-71b by predicting and verifying its target genes. The seeds of the miRNA area mainly exist in the target mRNA 3' UTR, while a few are also located in the 5' UTR and mRNA coding regions. Prediction of target mRNA using the miRanda software is largely based on the complementary matching of miRNA and target mRNA 3' UTRs, thermodynamic stability, and target seed area conservative principles. We used the constructed planaria 3' UTR database and miRanda software to predict numerous target genes for miR-71b. Based on the score, three target genes of miR-71b were selected: TNF receptor-associated factor-2-like protein A (TRAF-2A), heat shock protein 70 ( $h s p 70)$, and age-1.

To identify target mRNA genes of miR-71b, we examined whether the expression of any mRNAs was upregulated upon inhibition of miR-71b function compared with expression in the control group. The three target mRNAs (TRAF-2A, hsp70, and age-1) were observed to be upregulated at least 1.8-fold in the experimental individuals (Figure 4). In addition, the daf-16 gene (FOXO transcription factor), which is the downstream product of age-1 in the IIS pathway rather than a predicted target gene of miR-71b, was reduced 0.02-fold upon inhibition of miR-71b function (Figure 4). $\beta$-actin and $D j e f_{2}$ were used as separate control genes, which yielded the same results (Figure 4).

(A)

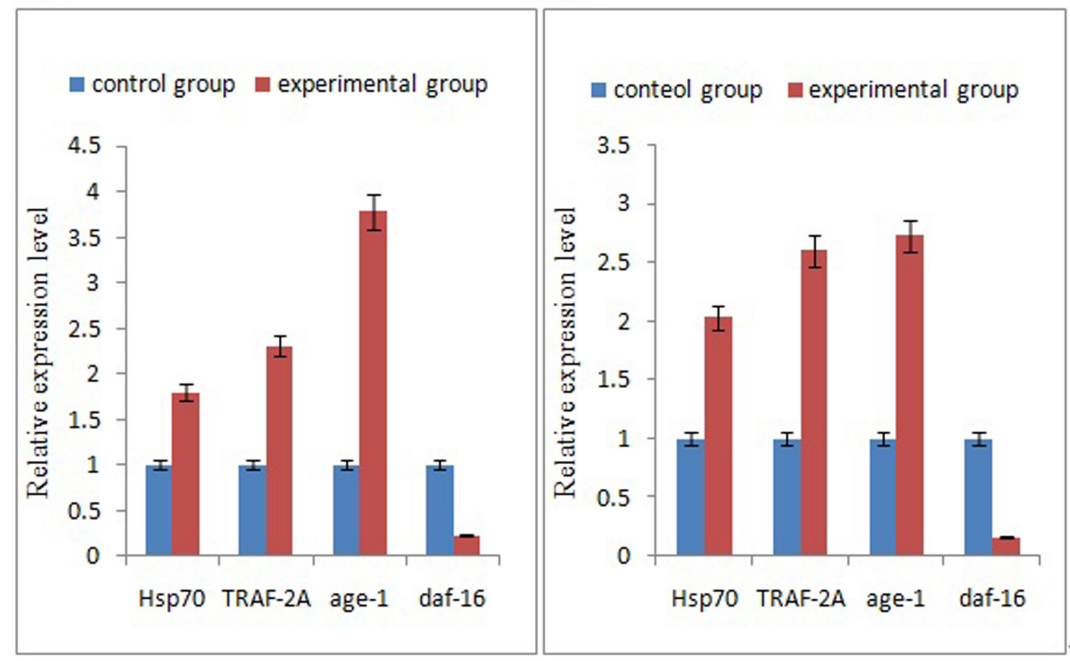

Figure 4. Relative expression of miR-71b target genes in planarians under control conditions (injected with RNase free water) and under loss of function conditions (experimental group, injected with antagomir71b). A. $\beta$-actin used as a control gene, B. Djef2 used as a control gene.

\section{DISCUSSION}

\section{Expression analysis of miR-71b and target genes over time}

The regulation of miRNAs and their target mRNAs occurs in many biological processes, such as differentiation, apoptosis, proliferation, and cell-fate determination (Hornstein, 2010; Mann et al., 2010). In this study, we focused on investigating the effects of miRNA loss of function. To 
increase the binding affinity of miRNAs and to improve nuclease resistance and in vivo delivery, antisense oligonucleotides (anti-miRs) are required in a variety of chemical modifications (Stenvang and Kauppinen, 2008; Lennox and Behlke, 2011). In this study, we used antagomirs to inhibit the function of miR-71b.

We studied starvation-induced planarians, in which neoblast progeny numbers decreased during starvation, by analyzing the levels of neoblast expression and the neoblast progeny markers using qPCR at different starvation periods (González-Estévez et al., 2012). Specifically, we assessed the expression of miR-71b at starvation periods of $0 \mathrm{~h}$, and 7, 14, and 21 days using qPCR. We found that expression of miR-71b was upregulated during starvation, suggesting that miR-71b has an important function in starvation-induced planarians.

\section{Tissue-specific expression of $\mathrm{miR}-71 \mathrm{~b}$}

Tissue-specific expression of miR-71b was detected in planarians using WISH. Formaldehyde-based whole-mount in situ hybridization was performed on the planarians on the basis of the protocols originally described by Pearson et al. (2009). miR-71b was clearly expressed in the pharynx and brain ganglia of the planarians. Spatial expression patterns of miR-71b have been located in the parenchyma (an organ for feeding and excretion) and brain ganglia of Schmidtea mediterranea and D. japonica (González-Estévez et al., 2009). The results of studies in these species indirectly explain the important function of miR-71b in starvation-induced planarians.

\section{Research on the function of miR-71b}

The IIS pathway has critical roles in the control of longevity, development, and metabolism across phylogeny. In addition, miR-71 is an effective regulator of the long-term survival and recovery of $C$. elegans from starvation-induced L1 diapause due to its functional relationship with the IIS pathway. The age-1 gene, which is the gene of the IIS pathway, was predicted to be a target gene of miR-71, using TargetScan and mirWIP, and validated as a miR-71 target gene using a dual-color 3' UTR reporter system (Zhang et al., 2011).

Daf-16 is a FOXO transcription factor acting downstream of age-1 in the IIS pathway. If the expression of age-1 was upregulated, the FOXO transcription factor would be critically and negatively downregulated (Lewis et al., 2005). By using the search function of miRBase, we determined that the miR-71b of $D$. japonica and miR-71 of $C$. elegans are members of one miRNA family. These genes have identical seed regions that anchor the miRNA to the mRNA target. Therefore, different miRNAs can possess the same target gene, and different genes can be regulated by the same miRNA

We hypothesized that miR-71b may play important roles in $D$. japonica. To validate our hypothesis, we examined the interaction among miR-71b, age-1, and daf-16 in planarians. We found increased expression of age-1 and decreased expression of daf-16 following the loss of miR-71b function, suggesting that the insulin receptor pathway may also play a role in $D$. japonica, particularly in starvation-induced individuals. This finding explains why miR-71b expression is high in planarians starved for 7 days.

In our laboratory, small non-coding RNAs, particularly miRNAs, were identified in $D$. japonica using deep sequencing, and we found that some of the expression patterns of planarian miRNAs differed greatly between regenerative and normal tissues (Qin et al., 2012). We also utilized a tag-based digital gene expression system to research transcriptome changes during 
different periods of regeneration (Qin et al., 2011). Based on these experimental results and the results of the miRanda software used in the current study, we determined that TRAF-2A and $h s p 70$ are the target genes of miR-71b. Upon interference of miR-71b with antagomir71b, the expression of $T R A F-2 A$ and $h s p 70$ was observed to increase, similar to the age- 1 gene. This finding suggests that TRAF-2A, $h s p 70$, and age-1 are all target genes of miR-71b in $D$. japonica.

In summary, we have demonstrated that miR-71b can be effectively and sequencespecifically inhibited with antagomir. The above result demonstrates that injection with antagomir is an effective method to reduce miRNA expression in planarians. This technique will be valuable for therapeutic intervention to control miRNA activity in disease states. Furthermore, we linked the miRNA with the signaling pathway in planarians and showed that miR-71b plays an important role in the development of starvation-induced planarians.

\section{Conflicts of interest}

The authors declare no conflict of interest.

\section{ACKNOWLEDGMENTS}

Research supported by the National Natural Science Foundation of China (\#31207384) and the Program for Science \& Technology Innovation Talents in Universities of Henan Province (\#13HASTIT023).

\section{REFERENCES}

Baguñà J, Saló E and Romero R (1989). Effects of activators and antagonists of the neuropeptides substance $P$ and substance K on cell proliferation in planarians. Int. J. Dev. Biol. 33: 261-266.

Barbieri M, Bonafè M, Franceschi C and Paolisso G (2003). Insulin/IGF-I-signaling pathway: an evolutionarily conserved mechanism of longevity from yeast to humans. Am. J. Physiol. Endocrinol. Metab. 285: E1064-E1071.

Braasch DA and Corey DR (2001). Locked nucleic acid (LNA): fine-tuning the recognition of DNA and RNA. Chem. Biol. 8: 1-7.

Chen C, Ridzon DA, Broomer AJ, Zhou Z, et al. (2005). Real-time quantification of microRNAs by stem-loop RT-PCR. Nucleic Acids Res. 33: e179.

Enright AJ, John B, Gaul U, Tuschl T, et al. (2003). MicroRNA targets in Drosophila. Genome Biol. 5: R1.

González-Estévez C, Arseni V, Thambyrajah RS, Felix DA, et al. (2009). Diverse miRNA spatial expression patterns suggest important roles in homeostasis and regeneration in planarians. Int. J. Dev. Biol. 53: 493-505.

González-Estévez C, Felix DA, Rodríguez-Esteban G and Aboobaker AA (2012). Decreased neoblast progeny and increased cell death during starvation-induced planarian degrowth. Int. J. Dev. Biol. 56: 83-91.

Hornstein E (2010). miRNA activity directs stem cell commitment to a particular fate. Cell Cycle 9: 4041-4042.

Koshkin AA, Singh SK, Nielsen P, Rajwanshi VK, et al. (1998). LNA (locked nucleic acids): synthesis of the adenine, cytosine, guanine, 5-methylcytosine, thymine and uracil bicyclonucleoside monomers, oligomerisation, and unprecedented nucleic acid recognition. Tetrahedron 54: 3607-3630.

Krek A, Grun D, Poy MN, Wolf R, et al. (2005). Combinatorial microRNA target predictions. Nature Genet. 37: 495-500.

Lennox KA and Behlke MA (2011). Chemical modification and design of anti-miRNA oligonucleotides. Gene Ther. 18: 1111-1120.

Lewis BP, Shih IH, Jones-Rhoades MW, Bartel DP, et al. (2003). Prediction of mammalian microRNA targets. Cell 115: 787-798.

Lewis BP, Burge CB and Bartel DP (2005). Conserved seed pairing, often flanked by adenosines, indicates that thousands of human genes are microRNA targets. Cell 120: 15-20.

Livak KJ and Schmittgen TD (2001). Analysis of relative gene expression data using real-time quantitative PCR and the 2(-Delta Delta C (T)) Method. Methods 25: 402-408.

Mann M, Barad O, Agami R, Geiger B, et al. (2010). miRNA-based mechanism for the commitment of multipotent progenitors to a single cellular fate. Proc. Natl. Acad. Sci. U. S. A. 107: 15804-15809.

Michelangela B, Massimiliano B, Claudio F, Paolisso G, et al. (2003). Insulin/IGF-I-signaling pathway: an evolutionarily conserved mechanism of longevity from yeast to humans. Am J. Physiol. Endocrinol. Metab. 285: E1064-1071.

Miranda KC, Huynh T, Tay Y, Ang YS, et al. (2006). A pattern-based method for the identification of microRNA binding sites 
and their corresponding heteroduplexes. Cell 126: 1203-1217.

Narasimhan SD, Yen K and Tissenbaum HA (2009). Converging pathways in lifespan regulation. Curr. Biol. 19: R657-R666. Newmark PA and Sánchez Alvarado A (2002). Not your father's planarian: a classic model enters the era of functional genomics. Nat. Rev. Genet. 3: 210-219.

Obika S, Nanbu D, Hari Y, Andoh J, et al. (1998). Stability and structural features of the duplexes containing nucleoside analogues with a fixed N-type conformation, 2'-O,4'-C-methyleneribonucleosides. Tetrahedron Lett. 39: 5401-5404.

Pearson BJ, Eisenhoffer GT, Gurley GA, Rink JC, et al. (2009). Formaldehyde-based whole-mount in situ hybridization method for planarians. Dev. Dyn. 238: 443-450.

Petersen M and Wengel J (2003). LNA: a versatile tool for therapeutics and genomics. Trends Biotechnol. $21:$ 74-81.

Qin YF, Fang HM, Tian QN, Bao ZX, et al. (2011). Transcriptome profiling and digital gene expression by deep-sequencing in normal/regenerative tissues of planarian Dugesia japonica. Genomics 97: 364-371.

Qin YF, Zhao JM, Bao ZX, Zhu ZY, et al. (2012). Identification of small non-coding RNAs in the planarian Dugesia japonica via deep sequencing. Genomics 99: 315-321.

Rehmsmeier M, Steffen P, Hochsmann M and Giegerich R (2004). Fast and effective prediction of microRNA/target duplexes. RNA 10: 1507-1517.

Sri Devi N, Kelvin Y and Heidi A (2009).Converging pathways in lifespan regulation. Curr. Biol 19: 657-666.

Stenvang J and Kauppinen S (2008). MicroRNAs as targets for antisense-based therapeutics. Expert Opin. Biol. Ther. 8: 59-81.

Wagner DE, Wang IE and Reddien PW (2011). Clonogenic neoblasts are pluripotent adult stem cells that underlie planarian regeneration. Science 332: 811-816.

Zhang X, Zabinsky R, Teng Y, Cui M, et al. (2011). microRNAs play critical roles in the survival and recovery of Caenorhabditis elegans from starvation-induced L1 diapause. Proc. Natl. Acad. Sci. U. S. A. 108: 17997-8002. 